\title{
Wavelength Modulated Raman Spectroscopy for Biomedical Applications
}

\author{
Sebastian Dochow ${ }^{1}$, Norbert Bergner ${ }^{1}$, Christoph $\mathrm{Krafft}^{1}$, Joachim Clement ${ }^{2}$, \\ Michael Malizu $^{3}$, Bavishna Balagopal ${ }^{3}$, Rob Marchington ${ }^{3}$, Kishan Dholakia $^{3}$, Jürgen Popp ${ }^{1,4}$ \\ ${ }^{1}$ Institute of Photonic Technology, Albert Einstein Straße 9, 07745 Jena, Germany, \\ ${ }^{2}$ University Hospital - Friedrich-Schiller-University of Jena, Clinic for Internal Medicine I \\ Erlanger Allee 101, 07747 Jena, Germany \\ 3 University of St. Andrews, School of Physics and Astronomy, United Kingdom \\ ${ }^{4}$ Institute of Physical Chemistry and Abbe School of Photonics-Friedrich Schiller University \\ of Jena, Helmholtzweg 4, 07743 Jena, Germany
}

\section{Introduction}

Wavelength modulated Raman spectroscopy has recently been shown to suppress auto fluorescence backgrounds. The approach modulates the laser excitation wavelength with a frequency by a certain wavelength interval. Continuously collected spectra are processed by principal component analysis (PCA) to give the Raman signatures. The resulting demodulated spectra can be used as input for classification without further pre-processing. Additionally this technique allows acquiring Raman spectra under room light conditions. Therefore, the approach is a promising candidate for subsequent in-vivo applications such as optical biopsies and tumour margin detection.

\section{Methods}

This technique is applied to a model system of circulating tumour cells that consists of leukocytes from patient's blood, acute myeloid leukaemia cells (AML-OCI3), and breast tumour cells BT20 and MCF7. Altogether 780 cells were studied and forty modulated spectra were collected per cell. The data set was divided into $80 \%$ training spectra and $20 \%$ test spectra. Classifications using support vector machines were compared for single modulated spectra, mean modulated spectra and demodulated spectra after PCA-processing. The stability of classification was tested by performing and validating 200 times with randomly selected data sets.

\section{Results and Discussion}

Classification rates ranged from $80 \%$ for the validation set to $97 \%$ for the training set. The largest overlap was observed between breast cancer cell lines BT20 and MCF7. A three class model with both breast cancer cells combined improved the classification rates to better than $97 \%$. The lower rates based on demodulated suggests that the distinction of cells is affected by absence or presence of fluorescence background. The results are transferred to a new data set of vascular tissue collected using a fiber-optic Raman probe. 